\title{
TERAPIA FOCAL EN CÁNCER DE PRÓSTATA: UNA POTENCIAL ESTRATEGIA PARA SOLUCIONAR EL PROBLEMA DEL SOBRETRATAMIENTO
}

\author{
Emilie Lecornett,2, Hashim Uddin Ahmed', Caroline Moore' y Mark Emberton?3. \\ 'Division de Cirugía y Ciencias Intervencionistas. University College of London. Londres. \\ 2 Servicio de Urología. Hôpital Claude Huriez. Francia. \\ ${ }^{3}$ UCLH/UCL Comprehensive Biomedical Research Centre. Londres. Inglaterra.
}

Resumen.- La terapia focal pretende destruir el foco tumoral con un margen de tejido circundante para ofrecer a los pacientes el potencial de combinar control oncológico y mínimos efectos colaterales. El tratamiento estándar actual incluye vigilancia activa o tratamiento radical. Ninguna de estas opciones es ideal. La vigilancia activa conlleva el riesgo de infratratamiento, morbilidad psicológica como resultado de la ansiedad, y no está libre de efectos colaterales debido a las biopsias

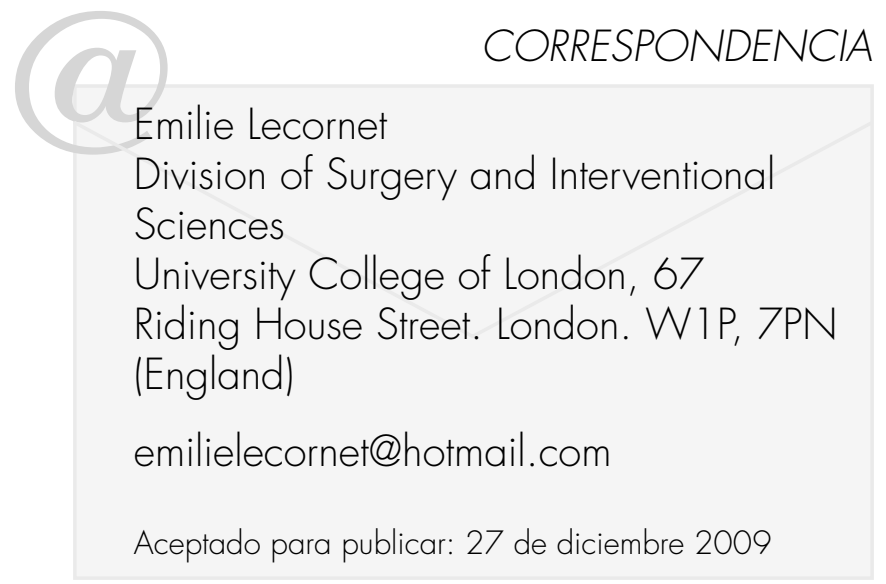

repetidas. Aunque el tratamiento radical es el patrón oro del tratamiento curativo, conlleva riesgos de sobretratamiento con sus numerosos efectos colaterales. Con el aumento de la proporción de cánceres unifocales o unilaterales entre varones de bajo riesgo, un abordaje focal podría evitar tanto el infra como el sobretratamiento. Con la mejora de la precisión de la localización del cáncer proporcionada por la resonancia magnética nuclear multiparamétrica y las nuevas estrategias de biopsia como los mapas biópsicos transperineales, las terapias ablativas como crioterapia, ultrasonidos focalizados de alta intensidad (HIFU), terapia fotodinámica y ablación radiointersticial de tumores hacen del tratamiento focal una posibilidad real.

Palabras clave: Terapia focal. Crioterapia. HIFU. Terapia fotodinámica. Cáncer de próstata.

Summary.- Focal therapy involves destroying the cancer focus and the tissue surrounding the cancer in order to offer patients the potential of combining cancer control with minimal side-effects. Current standard of care involve either surveillance or radical therapy. Neither of these is ideal. Active surveillance carries risk of undertreatment, psychological morbidity as a result of anxiety and is not without side-effects due to repeated biopsies., Although radical therapy is the gold standard for $\mathrm{Cu}^{-}$ rative treatment it carries risks of over-treatment with its numerous side-effects. With the proportion of unifocal or unilateral disease among men with low-risk disease rising, a focal approach could avoid both under and over-treatment. With the advent of improved accuracy for cancer localization provided by multi-parametric MRI and new biopsy strategies such as transperineal mapping biopsies, ablative modalities such as cryotherapy, 
high intensity focused ultrasound, photodynamic therapy and radio-interstitial tumour ablation make a focal treatments a real possibility.

Keywords: Focal therapy. Cryotherapy. HIFU. Photodynamic therapy. Prostate cancer.

\section{INTRODUCCIÓN}

La terapia focal para el cáncer de próstata localizado de bajo riesgo es una nueva estrategia terapéutica que ha sido añadida recientemente al arsenal de abordajes que pueden adoptarse. Se una a la vigilancia expectante, la vigilancia activa y los numerosos tratamientos radicales actualmente en práctica para el manejo del cáncer de próstata. Este artículo resume por qué las opciones estándar no son ideales. En segundo lugar, discutimos las nuevas mejoras en localización tumoral con RMN y biopsias, y la base de literatura actual disponible sobre tratamientos con terapias focales $(1,2)$. Finalmente, resumimos qué grupo de pacientes es el mejor para recibir terapia focal.

\section{Concepto de terapia focal}

Actualmente el tratamiento del cáncer de próstata localizado fluctua desde la vigilancia activa al tratamiento radical. Actualmente se detecta una proporción mayor de cáncer de próstata de riesgo bajo e intermedio debido al uso del cribado poblacional con PSA. Con frecuencia, los varones con cáncer de próstata localizado son jóvenes y tienen una expectativa de vida mayor de 15 años. La inmensa mayoría de los varones no están interesados en los resultados funcionales y de calidad de vida asociados tradicionalmente con el tratamiento radical pero a la vez quieren tener su cáncer controlado y no bajo vigilancia. Se añade a este problema la falta de datos a largo plazo de los protocolos de vigilancia activa junto con la incapacidad por el momento de asegurar a los pacientes que la vigilancia activa conlleva un riesgo bajo de progresión local o enfermedad metastática, punto en el que se pierde la ventana para la terapia curativa.

Por lo tanto, un tratamiento focal que destruya el foco cancerígeno con un pequeño margen de tejido normal alrededor puede ser una solución para estos pacientes.

Las características propias del cáncer de próstata hacen pensar a los médicos que el tratamiento focal sería suficiente para conseguir el benefi- cio del control oncológico evitando al mismo tiempo los daños colaterales del tratamiento radical. Hace tan sólo unos pocos años no había ninguna opción disponible entre la vigilancia activa y el tratamiento radical. En los últimos años se han evaluado y desarrollado diferentes técnicas de tratamiento focal. Esto ha sido posible en gran parte por las mejoras en la localización del cáncer, utilizando técnicas de imagen y de mapeo biópsico.

\section{Problemas del tratamiento estándar}

\section{Vigilancia Activa}

Los pacientes que cumplen los criterios para la vigilancia activa representan un grupo de muy bajo riesgo. Los criterios utilizados generalmente son PSA $<10 \mathrm{ng} / \mathrm{ml}$, tiempo de duplicación del PSA >3 años, Estadios T1c a T2a, Gleason $<7$, porcentaje de cilindros positivos y presencia del tumor $<50 \%$ de un solo cilindro (3-8). Sin embargo, estos criterios pueden no ser suficientemente estrictos si la prueba diagnóstica en que se basan tiene errores aleatorios y sistemáticos significativos. Por lo tanto, no es sorprendente que en biopsias repetidas se encontrará que 1 de cada 4 a 1 de cada 3 hombres inicialmente adecuado para vigilancia activa tenían un grado de Gleason superior o una mayor carga tumoral (9).

Además, aunque se piensa que la vigilancia activa no tiene efectos colaterales, al no haber tratamiento hasta la progresión, esta afirmación debería moderarse. La vigilancia activa puede entrañar un deterioro de la calidad de vida como muestran los cuestionarios de calidad de vida (10-13).

Deberíamos también considerar la carga psicológica de vivir con un cáncer no tratado, con la incertidumbre sobre la progresión de la enfermedad y la ansiedad, así como las cargas de cuidados y financieras asociadas con el intenso programa de vigilancia. Algunos grupos han intentado demostrar las consecuencias psicológicas de la vigilancia activa (14-16), encontrando unos niveles de ansiedad superiores en la vigilancia. Sin embargo, otros no han encontrado esto (17).

¿Podemos determinar si los pacientes bajo este régimen no desarrollaran un cáncer incurable durante el periodo de vigilancia? Se han comunicado casos de pacientes que se pensaba eran adecuados para vigilancia activa pero una vez sometidos a prostatectomía radical se encontró que tenían una enfermedad más agresiva y en algunos casos incurable (extensión extracapsular o metástasis ganglionares) $(13,18)$. Muchos han argumentado que debido 
a que tenemos una capacidad limitada para predecir qué cánceres pueden ser observados con seguridad sin que progresen (19), ningún hombre con cáncer de próstata debe ser sometido a vigilancia (20).

\section{Tratamiento radical}

Una vez diagnosticado un cáncer de próstata localizado el tratamiento radical sigue siendo el "gold standard". Sin embargo, los tratamientos radicales de la glándula completa pueden dar lugar a efectos colaterales significativos dañando o lesionando las estructuras vecinas, como la vejiga (especialmente el cuello vesical), las bandeletas neurovasculares, el esfínter externo y el recto. Esto puede conducir invariablemente a una reducción de la capacidad vesical, incontinencia de urgencia o estenosis de cuello vesical, estenosis de uretra, incontinencia de esfuerzo, impotencia y toxicidad intestinal (diarrea, sangrado, dolor). Cualquiera que sea el tratamiento elegido, existe una similitud en los efectos colaterales siendo su frecuencia dependiente de la modalidad de tratamiento empleada. Vivir con las secuelas del tratamiento podría ser potencialmente peor que vivir con la enfermedad, que podría no progresar nunca, o progresar tan lentamente que la mayoría de los pacientes murieran por otras causas (9). A pesar de las mejoras demostradas por la cirugía y la radioterapia durante los últimos años, por ejemplo con la cirugía laparoscópica o robótica, o la radioterapia de intensidad modulada, sus beneficios son limitados sin mejoras significativas en la calidad de vida después del tratamiento radical $(21,22)$.

\section{Técnicas de tratamiento focal}

\section{HIFU (High Intensity Focused Ultrasound): Ultrasoni- dos focalizados de alta energía}

La focalización estrecha de las ondas de ultrasonidos genera una alta densidad de energía. EI HIFU terapéutico se administra generalmente utilizando frecuencias bajas como $3,5-4,5 \mathrm{MHz}$, produciendo calor y "cavitación por inercia", y después destrucción tisular. Cuando la temperatura asciende a $56^{\circ} \mathrm{C}$ y se mantienen durante al menos 1 segundo puede ocurrir daño tisular, con el resultado de necrosis coagulativa y una respuesta inflamatoria. La temperatura alcanzada típicamente con HIFU es superior a $80^{\circ} \mathrm{C}$. Actualmente hay dos aparatos transrectales: El Ablatherm ${ }^{\circledR}$ (Edap-Technomed, Lyon, Francia) y el Sonoblate ${ }^{\circledR} 500$ (Focus surgery, Indianápolis, IN).

Datos de revisiones recientes sobre HIFU utilizado para la ablación completa de la glándula demuestran tasas de biopsias negativas a cinco años entre el $87 \%$ y el $97 \%(1,23,24)$, con tasas de supervivencia libre de enfermedad a cinco años que varían entre el $66 \%$ y el $78 \%$, de acuerdo con los criterios de ASTRO $(1,24)$. Los efectos secundarios comunicados en el abordaje completo de la glándula son incontinencia $(0,5 \%-15,4 \%)$, estenosis de uretra (24\%), fístula (0-2\%) e impotencia (13-53\%) (25). Sin embargo, estos efectos colaterales están mal comunicados (1). El HIFU es prometedor porque permite tratar lesiones con precisión y parece tener baja morbilidad. Además, podría asociarse la termografía por RMN para permitir monitorización de la temperatura durante el tratamiento.

\section{Crioterapia}

Las temperaturas muy bajas pueden producir numerosos efectos tóxicos en el tumor. La destrucción tisular se produce por lesión vascular, citolisis directa, formación de cristales de hielo, deshidratación intracelular, cambios de $\mathrm{pH}$, crioactivación de respuestas inmunes e inducción de la apoptosis. También produce daño endotelial que conduce a la agregación plaquetaria y microtrombosis. 3 minutos de congelación a una temperatura de $0^{\circ} \mathrm{C}$ puede producir ablación tisular eficaz. Las máquinas de crioterapia de $3^{\mathrm{a}} \mathrm{ge}$ neración ejecutan la ablación prostática bajo control por ecografía transrectal y con calentadores uretrales. Las pequeñas agujas utilizan gas a presión que congela y descongela el tejido. El tamaño de la aguja permite al cirujano realizar un tratamiento relativamente preciso, de forma que el daño a las estructuras vecinas puede minimizarse. Si embargo, la falta de control en tiempo real es un problema porque todo lo que el usuario visualiza es el frente hipoecoico de una bola de hielo que no representa el gradiente de temperaturas con precisión.

La crioterapia es la técnica de ablación terapéutica más estudiada. Ahmed y cols. Identificaron 13 series de crioterapia de las que se podían sacar datos, y encontraron que conforme a los criterios de ASTRO la crioterapia tiene un buen control bioquímico (25) cuando se trata la glándula al completo. Utilizando los criterios de Phenix, se encontró que había una supervivencia libre de recidiva bioquímica del $80,5 \%$ en los grupos de bajo riesgo. La supervivencia a 5 años fue $91,1 \%$. La tasa de biopsias negativa a los 10 años fue del $73,8 \%(26)$.

Los datos de crioterapia focal, aunque son más recientes, y por lo tanto limitados por un seguimiento corto, muestran supervivencias libres de recidiva bioquímica que varían entre el $80 \%$ y el $96 \%$ $(9,27-31)$ y supervivencias libres de enfermedad del $84 \%$ a tres años (26). Sin embargo, las comparaciones entre estudios son difíciles puesto que no existe 
una medida consensuada o validada de éxito o fracaso después del tratamiento focal.

La morbilidad parece disminuir con la crioterapia focal, con una superior calidad de vida relacionada con la salud debido a las menores tasas de incontinencia, fístulas o disfunción eréctil (32). Un estudio multicéntrico con 106 pacientes, utilizando aparatos de $3^{a}$ generación, comunicaba unas tasas de morbilidad en la crioterapia de la glándula completa del $5 \%$ de descamación de la uretra (Sloughing), 3\% de incontinencia con utilización de compresas, 5\% de incontinencia de urgencia sin compresas, $3,3 \%$ de retención urinaria transitoria, y $2,6 \%$ de dolor rectal. Los resultados contemporáneos de potencia después de crioterapia focal están entre $80-90 \%(26)$. Sin embargo todavía son necesarios datos a largo plazo. En un futuro próximo seremos capaces de mejorar la evaluación de crioterapia focal, como resultado del análisis de grandes bases de datos multicéntricas como el registro COLD (Cryo On-Line Data).

\section{Terapia Fotodinámica}

La terapia fotodinámica utiliza fármacos fotosensibilizantes que se acumulan preferentemente en algunos tejidos. Se activa después el fármaco con luz de una longitud de onda específica en el tejido o en los vasos. Para conseguir el efecto terapéutico es necesario el oxígeno tisular. Los fármacos activados, asociados con el oxígeno, crean daño tisular. Esta técnica está basada en el abordaje transperineal, utilizando un molde de braquiterapia para insertar las fibras ópticas que suministran luz laser de baja energía.

Se han publicado pocos estudios. Ahmed y cols. encontraron recientemente siete estudios (25). La eficacia de esta técnica parece prometedora, aunque son solo los resultados preliminares de los niveles de PSA después del tratamiento. En la mayoría de los estudios publicados no hay resultados de biopsias. Actualmente se están llevando a cabo ensayos clínicos para evaluar la técnica más a fondo. Los resultados preliminares son alentadores, pero es necesario introducir mejoras (33), y el control del cáncer tiene todavía que confirmarse (1).

\section{Ablación intersticial de tumores con radiofrecuencia: RITA (Radiofrequency Interstitial tumor ablation)}

Esta nueva tecnología se realiza por vía transperineal, utilizando agujas percutáneas que se insertan bajo control ecográfico. La energía de radiofrecuencia de bajo nivel calienta y destruye el tejido por necrosis coagulativa. No hay estudios de RITA en el tratamiento del cáncer de próstata y sólo unos pocos artículos históricos en su uso en hiperplasia benigna de próstata.

\section{La necesidad de una localización adecuada de la lesión}

Durante muchos años la técnica de imagen de la próstata fue la ecografía transrectal en modo B. Sin embargo, la ecografía transrectal tiene baja sensibilidad y especificidad en cáncer de próstata siendo otras patologías prostáticas como la hiperplasia benigna de próstata o las prostatitis también hipoecoicas. Las biopsias guiadas por ecografía transrectal solo pueden predecir la unilateralidad del cáncer en el $27,6 \%$ de los casos y esto no mejora añadiendo otras características de la biopsia como el porcentaje de tumor o el número de biopsias positivas (34). El número de biopsias necesario para localizar correctamente el cáncer es incierto. Algunos grupos han declarado que las biopsias guiadas por ecografía transrectal no son suficientes para la localización del tumor, o para establecer el estadiaje o el grado con precisión, en el caso de seleccionar pacientes para tratamiento focal (35-38).

En el momento actual se pueden realizar biopsias transperineales guiadas por ecografía. Utilizando un molde de braquiterapia y tomando una biopsia cada cinco milímetros se puede hacer un mapa de la extensión y localización del cáncer dentro de la próstata. Varios estudios han demostrado que las biopsias transperineales guiadas por ecografía demuestran con precisión el cáncer de próstata clínicamente significativo con un alto grado de sensibilidad (37). Las tasas de detección del cáncer aumentan desde el 29-34\% de las técnicas tradicionales al $47-70 \%$ de las biopsias transperineales $(39,40)$, particularmente en la parte anterior de la glándula que es inherentemente menos biopsiada $(35,37)$. La biopsia transperineal guiada por ecografía también permite determinar el grado de Gleason con precisión $(9,37,40)$. En modelos de simulación, la biopsia perineal guiada por ultrasonidos con punciones cada cinco milímetros podría detectar el 95\% de los cánceres significativos, definidos por un volumen de $0,5 \mathrm{~cm} 3$ (39). La toxicidad y la carga asistencial son las principales desventajas de las biopsias transperineales guiadas por ecografía. Aunque las biopsias perineales tienen una tasa de infección menor que las biopsias transrectales la tasa de retención es mayor, con algunos grupos que muestran tasas retención aguda de orina del $5-10 \%$ de incluso utilizando alfa bloqueantes perioperatorios (35).

Muchos grupos han concluido que las biopsias de mapeo debería recomendarse para la selec- 
ción de pacientes para tratamiento focal $(9,41,35)$ (36). Algunos también apuntan que puede haber dificultades para disección si se hace una prostatectomía radical posterior $(19,37,35)$.

Añadir Doppler color no parece mejorar la tasa de detección de tumores pequeños aunque su papel en tumores significativos puede ser útil (42). Recientemente, la ecografía transrectal con contraste se ha mostrado algo prometedora, con mayor sensibilidad en la detección de focos cancerígenos. Las tasas de detección de cáncer de próstata clínicamente significativo mejoraron en algunos estudios (43-46) con la habilidad de hacer biopsias dirigidas en tiempo real (42). El histoescaneado prostático (Prostate histoscanning) también se ha mostrado prometedor con una alta sensibilidad y especificidad para las lesiones significativas definidas por un volumen de 0,5 cc pero los estudios han sido pequeños y sin enmascaramiento. Se esperan los resultados de un estudio europeo multicéntrico con enmascaramiento actualmente en curso $(47,48)$.

Los protocolos de RMN multiparamétrica están mostrando actualmente resultados alentadores en la detección de lesiones significativas, con un volumen de corte de $0,5 \mathrm{~cm}^{3} \circ 0,2 \mathrm{~cm}^{3}$ y una localización precisa del cáncer así como su caracterización (grado, extensión extraprostática) $(49,43,50)$.

El tratamiento guiado por imagen, con ecografía con contraste $(51,52) \circ \operatorname{RMN}(53-56)$, está también en desarrollo. Sin embargo, el tratamiento guiado por RMN no es ideal actualmente por el entorno magnético, el problema del acceso a la RMN, el coste y la incomodidad para el paciente (43). En el momento presente, combinando los datos de imagen e histopatología de las biopsias de próstata de mapeo parece posible obtener la localización más precisa de las lesiones para aplicar tratamiento focal.

\section{Pacientes adecuados para terapia focal}

Por el momento no existe consenso sobre qué criterios deberían utilizarse para seleccionar pacientes para tratamiento focal. Con las actuales tendencias de cribado con PSA y el descenso del umbral de PSA para biopsia, del $45-85 \%$ de los pacientes entran en la categoría de bajo riesgo (PSA $<10 \mu g / L$, grado de Gleason 3+3, cT 1 c-cT2a). Se estima que entre el $25 \%$ y el $85 \%$ de los hombres que actualmente reciben tratamiento no morirían de la enfermedad si se les dejara sin tratamiento (enfermedad insignificante) (13).

El cáncer de próstata es visto generalmente como una enfermedad multifocal. Sin embargo, varios estudios, basándose en las piezas de prostatectomía radical, han encontrado una proporción significativa de hombres que tienen enfermedad unifocal o unilateral. Se ha comunicado que el cáncer de próstata es unilateral del $16 \%$ al $63 \%(9,21,38$, $57-60)$ y unifocal entre el $13 \%$ y el $26 \%(9,35,61)$. Este argumento conduce a la proposición de que en un número sustancial de hombres podría hacerse un abordaje de hemiablación de su enfermedad. Es interesante que un estudio reciente haya encontrado que los cánceres unifocales tienen un comportamiento más agresivo que la enfermedad multifocal. En una serie de 1159 prostatectomías radicales el estudio anatomopatológico encontró un 18,7\% de tumores Gleason 8-10 en los unifocales frente al 10,1\% en los multifocales. Además, hubo un $38,5 \%$ frente al $24,2 \%$ de recurrencia bioquímica, con una supervivencia libre de recidiva bioquímica significativamente peor en los cánceres unifocales.

La enfermedad multifocal no tiene necesariamente que ser excluida de la terapia focal. La evidencia apunta que los hombres con cáncer de próstata multifocal reúnen los requisitos para una forma de terapia focal si la ablación se dirige sólo a la/las lesión/es índice o significativas. Un punto de corte de 0,5 cc (diámetro menor de $10 \mathrm{~mm}$ ) puede utilizarse para predecir el riesgo de mortalidad a lo largo de la vida (62). El $80 \%$ de las lesiones secundarias son menores de $0,5 \mathrm{cc}(21,35,63$, 64). Además, se vio que los focos de cáncer secundarios tenían de media un volumen acumulativo menor de 0,3 cc. El 90\% de los casos de extensión extracapsular vienen de la lesión índice, y ésta representa el $80 \%$ de volumen total del tumor 113,2 $7,35,30,52,54,55,53,56,65,66)$. La presencia de focos cancerígenos secundarios y su volumen no tiene influencia sobre la recidiva bioquímica después de la prostatectomía radical (35). Por lo tanto, hay una pregunta de investigación importante e interesante sobre el tratamiento focal de la lesión índice y vigilancia de las lesiones secundarias no significativas (con la condición de que sean pequeñas y de grado de Gleason menor o igual a 6) (67). De hecho, está en marcha un ensayo clínico de este tipo en el Hospital de los autores (Protocolo registrado en el National Cancer Institute con el número NCT00988130).

Se han reunido algunos grupos de consenso para discutir las recomendaciones de terapia focal. En 2006 aparecieron los primeros criterios en la Conferencia de Consenso sobre Tratamiento Focal: Esperanza de vida $>5$ años, Estadio T1-T3, PSA $<15$ $\mathrm{ng} / \mathrm{ml}$, ausencia de enfermedad M1. Consideraron la afectación ganglionar como una contraindicación relativa. 
Pensaban que ni la densidad de PSA, ni el tiempo de duplicación del PSA, ni la puntuación de Gleason ni la ploidía debían tenerse en cuenta (36). Eggener y cols., en el grupo de trabajo TASK Force Group, propusieron los criterios para determinar qué pacientes deberían recibir terapia focal. Propusieron criterios que reflejaran los utilizados en vigilancia activa. La población en sí, discuten algunos, no debería tener ningún tratamiento. En otras palabras, estadios clínicos T1-T2a, PSA menor de $10 \mathrm{ng} / \mathrm{ml}$, densidad de PSA menor de 0,15 ng/ml, velocidad de PSA menos de $2 \mathrm{ng} / \mathrm{ml}$ anual, sin Gleason 4 o 5 , sin evidencia de extensión extraprostática y lesión única (68). Sartor y cols añaden que para definir las lesiones en RMN debería utilizarse una lesión con un diámetro mayor por técnicas de imagen $<15 \mathrm{~mm}$ en cualquier plano, que no exceda $5 \mathrm{~mm}$ de contacto con la cápsula en las imágenes axiales. Adicionalmente, los ganglios linfáticos regionales no deberían ser sospechosos de enfermedad metastásica. Con otras palabras, debería medir menos de $7 \mathrm{~mm}$ en el eje corto y tener un borde suave, y no debería ser un grupo asimétrico de nódulos (35).

\section{CONCLUSIONES}

La terapia focal en cáncer de próstata representa un cambio de perspectiva que puede suministrar el término medio entre infra-tratamiento y sobretratamiento ofreciendo la ablación del cáncer y la preservación del tejido no tumoral y las estructuras vitales circundantes para disminuir los efectos secundarios.

Avances recientes en la localización del cáncer con técnicas de imagen (RMN multiparamétrica, caracterización tisular con ecografía, ecografía con contraste) han demostrado unas tasas de precisión alentadoras en la detección de lesiones significativas así como en la localización del cáncer de próstata. Las biopsias transperineales guiadas por ecografía, que mapean la próstata cada $5 \mathrm{~mm}$, posiblemente suministran la precisión ideal en la localización y caracterización del cáncer para aplicar tratamiento focal aunque conllevan una carga asistencial. Son necesarios ensayos clínicos para evaluar la viabilidad, eficacia y seguridad de la terapia focal.

Muchos de estos ensayos clínicos están actualmente en marcha y si se verifican los signos tempranos de baja toxicidad temprana entonces es necesario planear estudios más grandes a largo plazo utilizando diseños de cohortes o diseños comparativos pragmáticos para evaluar la terapia focal en comparación con la vigilancia activa y los tratamientos radicales.

\section{Declaración de los autores}

Hashim Ahmed recibe fondos del Medical Research Council del Research Fellowship scheme. Hashim Ahmed y Mark Emberton reciben fondos de la Pelican Cancer Foundation, Reino Unido, The Prostate Research Campaign Reino Unido, St Peters Trust, Reino Unido y del Prostate Cancer Research Centre para la terapia focal. Mark Emberton también recibe fondos del UCLH/UCL Comprehensive Biomedical Research Centre. Además, Mark Emberton recibe fondos de investigación de Steba Biotech, Francia (Fabricantes de TOOKAD, un agente fotodinámico utilizado en tratamiento del cáncer de próstata) para ensayos clínicos de terapia focal. También es consejero medico de Focus Surgery/Misonix/USHIFU/ UKHIFU(Fabricantes y distribuidores del Sonablate ${ }^{\circledR}$ 500 HIFU). Mark Emberton es Director de Mediwatch PLC and Prostate Mapping Ltd.

Ninguna de las Fuentes de financiación ha participado en la escritura del presente artículo.

\section{BIBLIOGRAFÍA y LECTURAS RECOMENDADAS (*lectura de interés $y^{* *}$ lectura fundamental)}

1. Marberger M, et al. New treatments for localized prostate cancer. Urol, 2008; 72(6 Suppl): S36-43.

*2. Ahmed HU, et al. Will focal therapy become a standard of care for men with localized prostate cancer? Nat Clin Pract Oncol, 2007; 4(11):63242.

3. Klotz L. Active surveillance for prostate cancer: for whom? J Clin Oncol, 2005; 23(32): 8165-9.

4. Zhou P, et al. Predictors of prostate cancer-specific mortality after radical prostatectomy or radiation therapy. J Clin Oncol, 2005; 23(28): 6992-8.

5. Ahmed HU and Emberton M. Active surveillance and radical therapy in prostate cancer: can focal therapy offer the middle way? World J Urol, 2008; 26(5): 457-67.

6. Amin M, et al. Prognostic and predictive factors and reporting of prostate carcinoma in prostate needle biopsy specimens. Scand J Urol Nephrol Suppl, 2005; (216): 20-33.

7. Antunes AA, et al. The percentage of positive biopsy cores as a predictor of disease recurrence in patients with prostate cancer treated with radical prostatectomy. BJU Int, 2005; 96(9): 125863.

8. Freedland SJ, et al. Percent of prostate needle biopsy cores with cancer is significant independent predictor of prostate specific antigen recurrence following radical prostatectomy: results 
from SEARCH database. J Urol, 2003; 169(6): 2136-41.

9. Polascik TJ and Mouraviev V. Focal therapy for prostate cancer is a reasonable treatment option in properly selected patients. Urol, 2009; 74(4): 726-30.

10. Bacon CG, et al. The impact of cancer treatment on quality of life outcomes for patients with localized prostate cancer. J Urol, 2001; 166(5): 180410.

11. Galbraith ME, Ramirez JM, and Pedro LW. Quality of life, health outcomes, and identity for patients with prostate cancer in five different treatment groups. Oncol Nurs Forum, 2001; 28(3): 551-60.

12. Litwin MS, et al. Mental health in men treated for early stage prostate carcinoma: a posttreatment, longitudinal quality of life analysis from the Cancer of the Prostate Strategic Urologic Research Endeavor. Cancer, 2002; 95(1): 54-60.

13. Lindner $U$ and Trachtenberg J. Focal therapy for localized prostate cancer -choosing the middle ground. Can Urol Assoc J, 2009; 3(4): 333-335.

14. Latini DM, et al. The relationship between anxiety and time to treatment for patients with prostate cancer on surveillance. J Urol, 2007; 178(3 Pt 1): p. 826-31; discussion 831-2.

15. Dale $\mathrm{W}$, et al. The role of anxiety in prostate carcinoma: a structured review of the literature. Cancer, 2005; 104(3): 467-78.

16. Pickles T, et al. Psychosocial barriers to active surveillance for the management of early prostate cancer and a strategy for increased acceptance. BJU Int, 2007; 100(3): 544-51.

17. Burnet KL, et al. Does active surveillance for men with localized prostate cancer carry psychological morbidity? BJU Int, 2007; 100(3): 540-3.

*18. Klotz L. Active surveillance with selective delayed intervention for favorable risk prostate cancer. Urol Oncol, 2006; 24(1): 46-50.

19. Black P. There is no role for focal therapy in prostate cancer. Can Urol Assoc J, 2009; 3(4): 331332.

*20. Johansson JE, et al. Natural history of early, localized prostate cancer. JAMA, 2004; 291(22): 2713-9.

21. Jayram G and Eggener SE. Patient selection for focal therapy of localized prostate cancer. Curr Opin Urol, 2009; 19(3): 268-73.

22. Hu JC, et al. Patterns of care for radical prostatectomy in the United States from 2003 to 2005. J Urol, 2008; 180(5): 1969-74.

23. Thuroff $S$, et al. High-intensity focused ultrasound and localized prostate cancer: efficacy results from the European multicentric study. J Endourol, 2003; 17(8): 673-7.

24. Blana A, et al. Eight years' experience with high- intensity focused ultrasonography for treatment of localized prostate cancer. Urol, 2008; 72(6): 1329-33; discussion 1333-4.

25. Ahmed HU, Moore C, and Emberton M. Minimally-invasive technologies in uro-oncology: the role of cryotherapy, HIFU and photodynamic therapy in whole gland and focal therapy of localised prostate cancer. Surg Oncol, 2009; 18(3): 219-32.

26. Ritch CR and Katz AE. Prostate cryotherapy: current status. Curr Opin Urol, 2009; 19(2): 17781.

*27. Onik G. Rationale for a "male lumpectomy," a prostate cancer targeted approach using cryoablation: results in 21 patients with at least 2 years of follow-up. Cardiovasc Intervent Radiol, 2008; 31(1): 98-106.

28. Onik G, et al. The "male lumpectomy": focal therapy for prostate cancer using cryoablation results in 48 patients with at least 2-year follow-up. Urol Oncol, 2008; 26(5): 500-5.

*29. Bahn DK, et al. Focal prostate cryoablation: initial results show cancer control and potency preservation. J Endourol, 2006; 20(9): 688-92.

30. Ellis DS, Manny TB, and Rewcastle JC. Focal cryosurgery followed by penile rehabilitation as primary treatment for localized prostate cancer: initial results. Urol, 2007; 70(6 Suppl): 9-15.

31. Lambert EH, et al. Focal cryosurgery: encouraging health outcomes for unifocal prostate cancer. Urology, 2007; 69(6): 1117-20.

32. Ritch CR and Katz AE. Update on cryotherapy for localized prostate cancer. Curr Urol Rep, 2009; 10(3): 206-11.

33. Emberton M. VTP for men with prostate cancer - Early Results. J Endourol, 2008; 22: 3.

34. Tareen B, et al. Can contemporary transrectal prostate biopsy accurately select candidates for hemi-ablative focal therapy of prostate cancer? BJU Int, 2009; 104(2): 195-9.

35. Sartor AO, et al. Evaluating localized prostate cancer and identifying candidates for focal therapy. Urol, 2008: 72(6 Suppl): S12-24.

36. Bostwick DG, et al. Group consensus reports from the Consensus Conference on Focal Treatment of Prostatic Carcinoma, Celebration, Florida, February 24, 2006. Urol, 2007; 70(6 Suppl): 42-4.

*37. Onik G and Barzell W. Transperineal 3D mapping biopsy of the prostate: an essential tool in selecting patients for focal prostate cancer therapy. Urol Oncol, 2008; 26(5): 506-10.

38. Tsivian $\mathbf{M}$, et al. Predicting unilateral prostate cancer on routine diagnostic biopsy: sextant vs extended. BJU Int, 2009.

*39. Crawford ED, et al. Clinical staging of prostate cancer: a computer-simulated study of transperineal prostate biopsy. BJU Int, 2005; 96(7): 9991004. 
40. Turpen R and Rosser CJ. Focal therapy for prostate cancer: revolution or evolution? BMC Urol, 2009; 9: 2.

41. Barzell WE and Melamed MR. Appropriate patient selection in the focal treatment of prostate cancer: the role of transperineal 3-dimensional pathologic mapping of the prostate--a 4-year experience. Urol, 2007; 70(6 Suppl): 27-35.

42. Turkbey B, et al. Imaging localized prostate cancer: current approaches and new developments. AJR Am J Roentgenol, 2009; 192(6): 1471-80.

*43. Turkbey B, Pinto PA, and Choyke PL. Imaging techniques for prostate cancer: implications for focal therapy. Nat Rev Urol, 2009; 6(4): 191-203.

44. Yang JC, et al. Contrast-enhanced gray-scale transrectal ultrasound-guided prostate biopsy in men with elevated serum prostate-specific antigen levels. Acad Radiol, 2008; 15(10): 1291-7.

45. Tang J, et al. Peripheral zone hypoechoic lesions of the prostate: evaluation with contrast-enhanced gray scale transrectal ultrasonography. J Ultrasound Med, 2007; 26(12): 1671-9.

46. Halpern EJ, et al. Detection of prostate carcinoma with contrast-enhanced sonography using intermittent harmonic imaging. Cancer, 2005; 104(11): 2373-83.

47. Braeckman J, et al. Computer-aided ultrasonography (HistoScanning): a novel technology for locating and characterizing prostate cancer. BJU Int, 2008; 101(3): 293-8.

48. Braeckman $\mathbf{J}$, et al. The accuracy of transrectal ultrasonography supplemented with computer-aided ultrasonography for detecting small prostate cancers. BJU Int, 2008; 102(11): 1560-5.

49. Ahmed HU, et al. Is it time to consider a role for MRI before prostate biopsy? Nat Rev Clin Oncol, 2009; 6(4): 197-206.

50. Krieger A, et al. Design of a novel MRI compatible manipulator for image guided prostate interventions. IEEE Trans Biomed Eng, 2005; 52(2): 306-13.

51. Atri M, et al, Contrast-enhanced ultrasonography for real-time monitoring of interstitial laser thermal therapy in the focal treatment of prostate cancer. Can Urol Assoc J, 2009; 3(2): 125-30.

*52. Lindner U, et al. Image guided photothermal focal therapy for localized prostate cancer: phase I trial. J Urol, 2009; 182(4): 1371-7.

53. McNichols RJ, et al. MR thermometry-based feedback control of laser interstitial thermal therapy at $980 \mathrm{~nm}$. Lasers Surg Med, 2004; 34(1): 48-55.

54. de Senneville BD, Mougenot C, and Moonen CT. Real-time adaptive methods for treatment of mobile organs by MRI-controlled high-intensity focused ultrasound. Magn Reson Med, 2007; 57(2): 319-30.
55. de Senneville BD, et al. MR thermometry for monitoring tumor ablation. Eur Radiol, 2007; 17(9): 2401-10.

56. Mougenot $\mathrm{C}$, et al. Three-dimensional spatial and temporal temperature control with MR thermometry-guided focused ultrasound (MRgHIFU). Magn Reson Med, 2009; 61(3): 603-14.

57. Mouraviev V, et al. Analysis of laterality and percentage of tumor involvement in 1386 prostatectomized specimens for selection of unilateral focal cryotherapy. Technol Cancer Res Treat, 2007; 6(2): 91-5.

58. Mouraviev V, Polascik TJ, Pathological background and its clinical implications for focal therapy of early detected prostate cancer. Nat Clin Pract Urol., in press.

59. Mouraviev V, et al. Prostate cancer laterality as a rationale of focal ablative therapy for the treatment of clinically localized prostate cancer. Cancer, 2007; 110(4): 906-10.

60. Tareen B, et al. Appropriate candidates for hemiablative focal therapy are infrequently encountered among men selected for radical prostatectomy in contemporary cohort. Urol, 2009; 73(2): 351-4; discussion 354-5.

61. De Laet K, et al. Predicting tumour location in radical prostatectomy specimens: same-patient comparisons of 21-sample versus sextant biopsy. BJU Int, 2009; 104(5): 616-20.

62. Villers A, et al. Current status of MRI for the diagnosis, staging and prognosis of prostate cancer: implications for focal therapy and active surveillance. Curr Opin Urol, 2009; 19(3): 274-82.

63. Nelson BA, et al. Tumour volume is an independent predictor of prostate-specific antigen recurrence in patients undergoing radical prostatectomy for clinically localized prostate cancer. BJU Int, 2006; 97(6): 1169-72.

64. Villers A, et al. Multiple cancers in the prostate. Morphologic features of clinically recognized versus incidental tumors. Cancer, 1992; 70(9): 2313-8.

65. Iczkowski KA, et al. Preoperative prediction of unifocal, unilateral, margin-negative, and small volume prostate cancer. Urol, 2008; 71(6):116671.

66. Noguchi M, et al. Prognostic factors for multifocal prostate cancer in radical prostatectomy specimens: lack of significance of secondary cancers. J Urol, 2003; 170(2 Pt 1): 459-63.

67. Ahmed HU. The index lesion and the origin of prostate cancer. N Engl J Med, 2009; 361(17): 1704-6.

68. Eggener SE, et al. Focal therapy for localized prostate cancer: a critical appraisal of rationale and modalities. J Urol, 2007; 178(6): 2260-7. 